\title{
Protein Biosynthesis in Mitochondria: Past Simple, Present Perfect, Future Indefinite
}

\author{
S. A. Levitskii ${ }^{1}$, M. V. Baleva ${ }^{1}$, I. V. Chicherin ${ }^{1}$, I. A. Krasheninnikov ${ }^{1}$, and P. A. Kamenski ${ }^{1, a *}$ \\ ${ }^{1}$ Lomonosov Moscow State University, Faculty of Biology, 119234 Moscow, Russia \\ ${ }^{a}$ e-mail: peter@protein.bio.msu.ru \\ Received November 6, 2019 \\ Revised December 6, 2019 \\ Accepted December 9, 2019
}

\begin{abstract}
Mitochondria are obligate organelles of most eukaryotic cells that perform many different functions important for cellular homeostasis. The main role of mitochondria is supplying cells with energy in a form of ATP, which is synthesized in a chain of oxidative phosphorylation reactions on the organelle inner membrane. It is commonly believed now that mitochondria have the endosymbiotic origin. In the course of evolution, they have lost most of their genetic material as a result of genome reduction and gene transfer to the nucleus. The majority of mitochondrial proteins are synthesized in the cytosol and then imported to the mitochondria. However, almost all known mitochondria still contain genomes that are maintained and expressed. The processes of protein biosynthesis in the mitochondria - mitochondrial translation - substantially differs from the analogous processes in bacteria and the cytosol of eukaryotic cells. Mitochondrial translation is characterized by a high degree of specialization and specific regulatory mechanisms. In this review, we analyze available information on the common principles of mitochondrial translation with emphasis on the molecular mechanisms of translation initiation in the mitochondria of yeast and mammalian cells.
\end{abstract}

DOI: $10.1134 / \mathrm{S} 0006297920030013$

Keywords: mitochondria, translation, initiation, translation factor, initiation factor

Mitochondria are important organelles in almost all eukaryotic cells. Their main function is supplying cells with the energy, which is provided in the form of ATP synthesized in the reactions of oxidative phosphorylation. Mitochondria also participate in the biosynthesis of $\mathrm{FeS}$ clusters, metabolism of amino acids and nucleotides, synthesis of lipids and steroids, and regulation of programmed cell death [1]. According to the widely accepted endosymbiotic theory, mitochondria have originated exogenously from a prokaryotic precursor similar to modern alpha-proteobacteria [2]. Mitochondria are semiautonomous organelles - during billions of years of evolution, mitochondria of various groups of eukaryotes have lost a significant portion of genetic material of their precursor, preserving only a small fraction of their genome. Mitochondrial proteome is represented mostly by the proteins encoded in the nucleus, synthesized in the cytoplasm, and imported to the mitochondria [3].

Abbreviations: fMet-tRNA, formylmethionine-tRNA; mtIF2 and mtIF3, translation initiation factors 2 and 3, respectively; 5'-UTR, 5'-untranslated region of mRNA.

* To whom correspondence should be addressed.
Mitochondrial genomes of modern eukaryotes are usually significantly smaller than the bacterial ones and contain a relatively small number of genes. Due to a significant divergence, different groups of eukaryotes differ considerably in the mitochondrial genome composition and number of mitochondrial genes. The smallest mitochondrial genome ( $6000 \mathrm{bp})$ has been found in the malaria infectious agent Plasmodium falciparum, while the total size of the multichromosomal mitochondrial DNA of the carnation family plant Silene conica exceeds $10,000,000 \mathrm{bp}[4,5]$.

The mitochondrial genome of baker's yeast (Saccharomyces cerevisiae) contains ribosomal RNA genes, complete set of tRNA genes, and genes for eight proteins - ribosomal protein Var1p and seven hydrophobic core proteins of the electron-transport chain complexes [6]. Mammalian mitochondrial genomes contain genes coding for rRNAs, tRNAs, and 13 proteins, components of the oxidative phosphorylation complexes [7]. At the same time, mitochondrial genomes of jakobids (Protozoa) have the largest number of mitochondrial genes among the known organisms and include genes of several ribosomal proteins, four-subunit RNA poly- 
merase, a reduced form of transfer-messenger RNA, RNA component of RNase $\mathrm{P}$, and proteins involved in the oxidative phosphorylation chain assembly, as well as rRNA and tRNA genes [8]. A significant degree of evolutionary divergence is indicated by the fact that the number of mitochondrial genes in different eukaryotes does not correlate with the size of mitochondrial genome. In particular, human mitochondrial genome contains 37 genes and has a size of $16,569 \mathrm{bp}$, whereas $S$. cerevisiae mitochondria genome contains approximately the same number of genes but consists of $\sim 80,000 \mathrm{bp}$, i.e., has the size similar to that of the mitochondrial genome of the abovementioned jakobids that includes $\sim 70$ genes [6-8]. Mitochondria of different eukaryotes also display significant differences in the mechanisms of gene expression (transcription and translation).

In this review, we present the latest discoveries in the studies of protein biosynthesis in yeast and mammalian mitochondria with special emphasis on the mechanisms of the translation initiation regulation. Without trying to comprehensively review all the known facts on the mitochondrial translation, we discuss existing contradictions and recently introduced novel concepts in this field of the studies.

\section{PROTEIN BIOSYNTHESIS IN MITOCHONDRIA}

Protein biosynthesis is carried out by ribosomes molecular machines that translate genetic information encrypted in mRNA and catalyze formation of peptide bonds between amino acids [9]. A ribosome id formed by the small and large subunits. The small subunit decodes mRNA, and the large subunit catalyzes the synthesis of the polypeptide chain. Despite the common principles of mRNA decoding and protein synthesis, the structure of ribosomes and the mechanisms of ribosome functioning differ not only between prokaryotes and eukaryotes, but also between bacteria and archaea [10]. Similarly, significant differences between the ribosomal structure and translation regulatory mechanisms have been observed for the mitochondrial ribosomes of different groups of organisms. For a long time, mitochondrial translation had remained the terra incognita of molecular biology; from the moment of its discovery, it has been assumed that protein biosynthesis in the mitochondria is similar to the one in bacteria. However, functional and, to a greater degree, structural studies of the last decade have significantly promoted our understanding of mitochondrial translation [11-13]. It has become obvious that this process is characterized by certain features that distinguish it from similar processes in bacteria or cytosol of eukaryotic cells. Mitochondrial translation has been investigated most comprehensively in $S$. cerevisiae and mammalian cells.

At present, bacterial origin of mitochondrial ribosomes causes no doubts [14]. In the process of evolution, mitochondrial ribosomes have significantly diverged from their bacterial precursors in both spatial structure and composition of ribosomal RNAs and ribosomal proteins. Explosive methodological development of cryo-electron microscopy in recent years has led to the resolving the structures of mitochondrial ribosomes from many organisms (Trypanosoma brucei [15], S. cerevisiae [16], Sus scrofa [12], Homo sapiens [11], Arabidopsis thaliana [17], and Brassica oleracea var. botrytis [18]). Comparison of these structures has revealed that even if mitoribosomes from different groups of eukaryotes differ significantly, they exhibit some common features. First of all, in comparison to the bacterial or cytoplasmic ribosomes, mitochondrial ribosomes are characterized by a significantly higher protein content (relative to RNA), often carry insertions and deletions in rRNA, and possess a specific set of proteins. Most likely, this is related to the partial replacement of RNA functions by the proteins, as well as to the fact that mitochondria synthesize only a limited number of hydrophobic proteins that are incorporated into the inner mitochondrial membrane as components of the oxidative phosphorylation complexes [19]. Protein biosynthesis in the mitochondria is strictly coupled with the synthesis of mitochondrial proteins in the cytoplasm, which is essential for correct assembly of the oxidative phosphorylation complexes [20]. Below, we present the data on the translation regulation in the mitochondria in yeast and mammalian cells (as the two most investigated systems) at the stage that is most susceptible to regulation, i.e., translation initiation.

\section{PAST SIMPLE}

As mentioned above, mitochondrial translation has a bacterial origin, which leads to the logical suggestion that protein biosynthesis initiation in the mitochondria should be somewhat similar to that in eubacteria. Initiation of bacterial translation has been investigated in great detail. The process starts with the formation of the $30 \mathrm{~S}$ translation initiation complex in which the start codon of mRNA interacts with the CAU anticodon of the initiator formylmethionine-tRNA (fMet-tRNA) at the P-site of the small ribosomal subunit. Three initiation factors (IF1, IF2, and IF3) play the most important role in the initiation complex formation; each of these proteins is essential for accurate and efficient translation initiation. All three factors interact with the corresponding sites on the $30 \mathrm{~S}$ subunit and perform their specific functions. IF2 in a complex with GTP binds fMet-tRNA, thus forming a ternary complex that interacts with the $30 \mathrm{~S}$ subunit. IF1 binds to the $30 \mathrm{~S}$ subunit and prevents the access of aminoacyl-tRNA to the A-site. It also increases the affinity of the IF2/GTP/fMet-tRNA ternary complex to the $30 \mathrm{~S}$ subunit. IF3 binds to the $30 \mathrm{~S}$ subunit and prevents its association with the $50 \mathrm{~S}$ subunit, as well as enables the 
accuracy of recognition of the fMet-tRNA start codon in the P-site. The complex of 30S with IF1, IF3, and IF2/GTP/fMet-tRNA binds short polypurine sequence in the $5^{\prime}$-untranslated region $\left(5^{\prime}\right.$-UTR) of mRNA (Shine-Dalgarno sequence) due to the presence of partially complementary sequence in $16 \mathrm{~S}$ rRNA. This binding is followed by the recognition of the start codon by the fMet-tRNA anticodon, GTP hydrolysis to GDP, dissociation of the initiation factors, and association of the small and large ribosomal subunits. The resulting 70S initiation complex represents an associated ribosome with the fMet-tRNA positioned in the P-site on the start AUG codon of mRNA and the A-site containing the second codon that expects arrival of the corresponding aminoacyl-tRNA. The initiation factors IF1 and IF2 are considered universal and highly conserved, as their functional and structural homologs have been found in all investigated bacteria and archaea [21].

Translation initiation in the mitochondria and, especially, the role and functions of mitochondrial initiation factors, have been studied to a much lesser extent. In particular, mitochondria of all investigated organisms lack IF1, while IF2 is absolutely universal and IF3 is almost universal [22]. It is likely that the mitochondrial initiation factor 2 (mtIF2) plays a key role in the translation initiation in mitochondria, and its functions will be discussed in detail further in this review.

\section{PRESENT PERFECT}

Bacterial IF2 is a GTPase consisting of six domains (I-VI), where domain IV hydrolyzes GTP and domain VI interacts directly with fMet-tRNA [23, 24]. IF2 selects the initiator aminoacyl-tRNA and facilitates association of ribosomal subunits during translation initiation (the latter function is evolutionary conserved in archaea and eukaryotes). Unlike bacteria, mammalian mitochondria do not have specialized methionine tRNA for the translation initiation but utilize the same tRNA used for methionine incorporation during elongation [25]. A portion of Met-tRNAs is formylated by a special enzyme; the resulting fMet-tRNA has a high affinity to mtIF2 and low affinity to the mitochondrial elongation factor EF-Tu. This dual function of Met-tRNA has been also found for T. brucei mitochondria which do not encode tRNAs required for the mitochondrial translation but import them from the cytoplasm [26].

It has been shown that human mtIF2 can functionally replace not only IF2, but also both IF2 and IF1 in Escherichia coli. Moreover, it has been demonstrated that the function of IF1 is carried out by a relatively small unique 37-a.a. domain located between the domains II and III in mtIF2 [27, 28]. The structure of the mtIF2 complex with porcine mitochondrial ribosome was resolved by Kummer et al. [29] by cryo-electron microscopy. The authors confirmed that the abovementioned 37-a.a. domain was located in the A-site of the small subunit. It prohibited the transfer of aminoacyltRNA to this site and prevented ribosome sliding over mRNA, i.e., functioned similarly to the bacterial IF1. Furthermore, the authors have been able to show that mtIF2 does not directly interact with mRNA and does not participate in its recruitment to the ribosome. It was established for the COX3 mRNA that the pentatricopeptide repeat (PPR)-containing mitoribosomal protein $\mathrm{mS} 39$ located in the region of the mRNA entry tunnel participated in the mtIF2 association with the mitoribosome [29]. Hence, it was suggested that mRNA interaction with the mitochondrial ribosome is provided by either individual mitoribosomal proteins or specialized translation factors.

Mitochondrial IF2 from $S$. cerevisiae encoded by the IFM1 gene has been investigated to a much lesser extent. Despite the fact that mIF2 was identified about 30 years ago [30], almost no structure-functional studies have been conducted on this protein. It was found that deletion of IFM1 results in the cessation of yeast growth on nonfermentable carbon sources [30]. It was also demonstrated in vitro that recombinant mIF2 forms a ternary complex with GTP and fMet-tRNA (or met-tRNA) which associates with the $30 \mathrm{~S}$ subunit of bacterial ribosome in the presence of synthetic mRNAs. Recombinant yeast mtIF2 was capable of GTP hydrolysis in the content of the complex, as well as prevented non-enzymatic hydrolysis of the indicated tRNAs [31]. Nevertheless, strict necessity of this factor for the translation initiation in the yeast mitochondria has not been demonstrated.

Bacterial translation initiation factor IF3 performs two important functions: it associates with the ribosomal 30S subunit following its dissociation after termination of translation, thus preventing association of the $30 \mathrm{~S}$ and $50 \mathrm{~S}$ subunits, and participates in the selection of tRNAs and mRNAs during initiation of a new round of protein synthesis by specifically destabilizing incorrectly formed codon-anticodon pairs [32, 33]. IF3 is universal and conserved in bacteria but is absent from the mitochondria of some organisms [22]. The functions of IF3 in the cytoplasm of eukaryotes are performed by the multisubunit factor eIF3 that does not display noticeable homology with bacterial IF3 [34].

Bacterial IF3 is a globular protein consisting of two well-defined $N$ - and $C$-terminal domains connected by an $\alpha$-helical linker [35]. The $C$-terminal domain interacts directly with 16S rRNA of the small ribosomal subunit, while the $N$-terminal domain can assume several conformations and provides interaction with fMet-tRNA [23]. Experiments on the deletion of IF3 fragments have demonstrated that isolated $C$-terminal domain could perform all functions characteristic for the full-size IF3, while the $N$-terminal domain provides additional strength to the IF3 binding to the ribosome and tRNA [36]. 
The amino acid sequence of mammalian mitochondrial translation initiation factor 3 (mtIF3) exhibits only limited homology to the sequence of the bacterial factor; however, these proteins are structurally similar, differing only in the presence of short $\mathrm{N}$ - and $\mathrm{C}$-terminal extensions in the mitochondrial factor [37]. In vitro studies have demonstrated that mammalian mtIF3 performs functions similar to those of the bacterial protein. In particular, mtIF3 is capable of dissociating mitochondrial ribosomes and facilitates formation of the initiation complex together with mtIF2 [37]. Similarly to the bacterial IF3, mammalian mtIF3 interacts with the mitoribosome predominantly via the $C$-terminal domain with some contribution of the linker sequence [38]. Moreover, mammalian mtIF3 also destabilizes the initiation complexes that do not contain mRNA or contain incorrect tRNA, although the latter activity is pronounced significantly less than in the bacterial IF3 $[39,40]$. The $N$ - and $C$-terminal extensions play an important role in the mtIF3 functioning. Thus, deletion of the $C$-terminal extension results in the inability of mtIF3 to dissociate incorrect initiation complexes, while deletion of the $\mathrm{N}$ terminal extension significantly increases the mtIF3 affinity to the 30 S subunit $[39,40]$. In 2019, Koripella et al. reported [41] the structure of the mammalian mtIF3 complex with the mitochondrial ribosome resolved by cryo-electron microscopy. It was found that in contrast to bacterial IF3, virtually all structural sites in mtIF3 participate in the interactions with the mitoribosome except the $C$-terminal extension. According to the modeling data, this structural element occupies the P-site during interaction of the mitoribosomal small subunit with the ternary mtIF2/GTP/fMet-tRNA complex in the absence of mRNA, thus preventing formation of the initiation complex without a template [41]. In the same study, the authors suggested the mtIF3 might be involved in the mRNA recruitment to the forming initiation complex.

For a long time, all attempts to identify the third translation initiation factor in $S$. cerevisiae mitochondria had been unsuccessful. However, recent studies have convincingly identified Aim23p protein as the mtIF3 in the yeast. The similarity between the ternary structures of Aim23p and mammalian mtIF3 was demonstrated using bioinformatics analysis; it was also found that the effect of the Aim23p gene deletion could be suppressed via expression of human and Schizosaccharomyces pombe mtIF3 proteins [22]. Aim 23p can associate with the small subunits of the yeast mitochondrial ribosome [42], as well as with the bacterial ribosome, causing their dissociation. Interestingly, in the case of bacterial ribosomes, this dissociation occurs via an unusual mechanism after binding to the associated $70 \mathrm{~S}$ ribosome, Aim23p interacts with both $30 \mathrm{~S}$ and $50 \mathrm{~S}$ subunits, facilitating formation of the intermediate dissociation state with the sedimentation coefficient of $\sim 60 \mathrm{~S}$ [43]. Both $N$ - and $C$ - terminal extensions of Aim23p have been found to be functionally important: deletion of these fragments results in the factor inactivation, while a hybrid protein obtained by addition of these sequences to IF3 from E. coli is fully active in the yeast mitochondria [44]. However, the most intriguing fact is that Aim23p is not essential for the mitochondrial translation. Deletion of the AIM23 gene results only in the reduced efficiency of COX1 and COX2 biosynthesis in the mitochondria, which phenotypically is manifested as slower adaptation of yeasts to the growth on non-fermentable carbon sources, but does not affect translation of other mitochondrial mRNAs [45]. This fact is quite surprising considering that the presence of IF3 is absolutely necessary for bacterial translation.

As mentioned above, most mitochondrial genes encode components of the oxidative phosphorylation chain. Therefore, mitochondrial translation should be coordinated with the cytosolic biosynthesis of the nuclear DNA-encoded subunits of these complexes. Regulation of mitotranslation remains poorly understood despite a significant progress achieved in the recent years. The mechanisms of translation regulation in $S$. cerevisiae mitochondria are the ones most extensively studied. A distinctive feature of this unique system is the presence of translational activators - groups of proteins that establish the efficiency of translation of one or another mRNA by interacting with the extended 5'-UTRs capable of forming the secondary structure. Translational activators have been discussed in detail in the recent reviews [46, 47].

The principles behind the regulation of biosynthesis of 13 proteins encoded in the mammalian mitochondrial genome, all of which are components of the oxidative phosphorylation chain complexes, are less obvious. First of all, this is due to the differences in the structure of mitochondrial mRNAs in mammals. These mRNAs lack the 5'-UTRs [48], which precludes an existence of translational activators similar to those found in yeast cells. So far, only one translational activator (TACO1) was identified in mammalian mitochondria, that determines the efficiency of cytochrome $c$ oxidase (COX1) biosynthesis; however, the mechanism of its action remains unknown [49].

Recent structural studies have shed the light on the mechanisms of recognition of mRNAs lacking the leader sequence by the mitochondrial ribosomes [29]. The structure of the initiation complex of mammalian mitochondrial ribosome with the COX3 mRNA assembled in vitro was resolved by cryo-electron microscopy. It has been established that translation initiation of this mRNA is mediated mostly by interaction of the PPR protein mS39 with the U-rich region after the seventh mRNA codon, which is conserved in all 11 mRNAs from the mammalian mitochondria [29]. These data, however, do not explain how the efficiency of translation initiation of any mRNA is determined. 


\section{FUTURE INDEFINITE}

To conclude our review, we will first summarize the data presented above. It had been assumed before that due to an indisputable origin of mitochondria from a bacterial precursor, mitochondrial translation is organized in a way similar to that in prokaryotes. However, the studies of recent 10-15 years have demonstrated that despite their common origin, the systems of protein biosynthesis in modern prokaryotes and mitochondria are very different, as well as the systems of mitochondrial translation in different groups of eukaryotes. First of all, these differences are manifested in the structure of mitochondrial ribosomes, which are specialized for the biosynthesis of mostly hydrophobic proteins encoded in the mitochondrial genome. The latter could explain the evolutionary origin of systems for the regulation of mitochondrial translation, the most studied of which involves translational activators in S. cerevisiae. The lack of similar system in mammalian mitochondria is due to significant differences in the mRNA structure (i.e., the absence of $5^{\prime}$-UTRs). Nevertheless, the existence of translation regulation in mammalian mitochondria is beyond doubts, because the biosynthesis of proteins encoded in the mitochondrial genome must be strictly coordinated with the biosynthesis of other components of the electron-transport chain in the cytosol. It is likely that one of the mechanisms of mitotranslation regulation involves coupling with the assembly of respiratory complexes in the inner mitochondrial membrane, although, this explanation is more relevant for the regulation of the elongation process, rather than translation initiation.

In this connection, of special interest are the data on the mitochondrial translation in yeast in the absence of the third initiation factor, which has little effect on the biosynthesis of the majority of mitochondrially-encoded proteins [45]. In essence, the profile of mitotranslation in the case of AIM23 deletion is similar to the one observed when a translational activator of a corresponding mRNA (e.g., Pet111p, an activator of the COX2 mRNA) is absent [50]. Based on these data, we suggest that mitochondrial evolution resulting in the loss of a majority of ancestral genes and mitotranslation specialization for the synthesis of a very limited number of hydrophobic proteins, as well as the need for a strict regulation of translation, have caused initiation factors to lose their versatility and become regulators of biosynthesis of individual proteins. Apparently, this hypothesis requires verification, for example, by investigating mitochondrial translation in the cells lacking genes of mitochondrial translation initiation factors. At the same time, it does not contradict presently available data, because all structural and functional studies of initiation factors have been performed in vitro, and the only in vivo model - absence of Aim 23p demonstrated that the third translation initiation factor is not essential for the protein biosynthesis in the mito- chondria. Apparently, this observation could be an exception characteristic only for the yeast system and this particular factor. However, it is our opinion that the question whether translation initiation factors are necessary for the biosynthesis of all mitochondrial proteins should be elucidated experimentally. Currently, these experiments are underway in our laboratory. Our hypothesis is corroborated in part by the recently reported data by Rudler et al. [51] who have demonstrated that deletion of mouse mtIF3 gene (Aim23p ortholog) does not abolish protein synthesis in the mitochondria but results only in its quantitative imbalance.

Funding. This work was supported by the Russian Science Foundation (project 17-14-01005; testing hypotheses on the evolution of mitochondrial translation apparatus) and Russian Foundation for Basic Research (project 19-14-50206).

Conflict of interest. The authors declare no conflict of interest in financial or any other sphere.

Ethical approval. This article does not contain description of studies with human participants or animals performed by any of the authors.

Open access. This article is distributed under the terms of the Creative Commons Attribution 4.0 International License (http://creativecommons.org/ licenses/by/4.0/), which permits unrestricted use, distribution, and reproduction in any medium, provided you give appropriate credit to the original author(s) and the source, provide a link to the Creative Commons license, and indicate if changes were made.

\section{REFERENCES}

1. Spinelli, J. B., and Haigis, M. C. (2018) The multifaceted contributions of mitochondria to cellular metabolism, Nat. Cell Biol., 20, 745-754.

2. Gray, M. W. (2012) Mitochondrial evolution, Cold Spring Harb. Perspect. Biol., 4, a011403; doi: 10.1101/cshperspect. a011403.

3. Gray, M. W. (2015) Mosaic nature of the mitochondrial proteome: implications for the origin and evolution of mitochondria, Proc. Natl. Acad. Sci. USA, 112, 1013310138; doi: 10.1073/pnas.1421379112.

4. Feagin, J. E., Harrell, M. I., Lee, J. C., Coe, K. J., Sands, B. H., Cannone, J. J., Tami, G., Schnare, M. N., and Gutell, R. R. (2012) The fragmented mitochondrial ribosomal RNAs of Plasmodium falciparum, PLoS One, 7, e38320; doi: 10.1371/journal.pone.0038320.

5. Sloan, D. B., Alverson, A. J., Chuckalovcak, J. P., Wu, M., McCauley, D. E., Palmer, J. D., and Taylor, D. R. (2012) Rapid evolution of enormous, multichromosomal genomes in flowering plant mitochondria with exceptionally high mutation rates, PLoS Biol., 10, e1001241; doi: 10.1371/ journal.pbio. 1001241 .

6. Freel, K. C., Friedrich, A., and Schacherer, J. (2015) Mitochondrial genome evolution in yeasts: an all-encom- 
passing view, FEMS Yeast Res., 15, fov023; doi: 10.1093/ femsyr/fov023.

7. Taanman, J. W. (1999) The mitochondrial genome: structure, transcription, translation and replication, Biochim. Biophys. Acta, 1410, 103-123.

8. Burger, G., Gray, M. W., Forget, L., and Lang, B. F. (2013) Strikingly bacteria-like and gene-rich mitochondrial genomes throughout jakobid protists, Genome Biol. Evol., 5, 418-438; doi: 10.1093/gbe/evt008.

9. Finkelstein, A. V., Razin, S. V., and Spirin, A. S. (2018) Intersubunit mobility of the ribosome, Mol. Biol. (Moscow), 52, 921-934.

10. Eme, L., Spang, A., Lombard, J., Stairs, C. W., and Ettema, T. J. G. (2017) Archaea and the origin of eukaryotes, Nat. Rev. Microbiol., 15, 711-723.

11. Amunts, A., Brown, A., Toots, J., Scheres, S. H. W., and Ramakrishnan, V. (2015) The structure of the human mitochondrial ribosome, Science, 348, 95-98; doi: 10.1126/ science.aaa1193.

12. Greber, B. J., Bieri, P., Leibundgut, M., Leitner, A., Aebersold, R., Boehringer, D., and Ban, N. (2015) The complete structure of the $55 \mathrm{~S}$ mammalian mitochondrial ribosome, Science, 348, 303-308; doi: 10.1126/science. aaa3872.

13. Englmeier, R., Pfeffer, S., and Forster, F. (2017) Structure of the human mitochondrial ribosome studied in situ by cryoelectron tomography, Structure, 25, 1574-1581.e2; doi: 10.1016/j.str.2017.07.011.

14. Bieri, P., Greber, B. J., and Ban, N. (2018) High-resolution structures of mitochondrial ribosomes and their functional implications, Curr. Opin. Struct. Biol., 49, 44-53.

15. Ramrath, D. J. F., Niemann, M., Leibundgut, M., Bieri, P., Prange, C., Horn, E. K., Leitner, A., Boehringer, D., Schneider, A., and Ban, N. (2018) Evolutionary shift toward protein-based architecture in trypanosomal mitochondrial ribosomes, Science, 362; doi: 10.1126/science. aau7735.

16. Desai, N., Brown, A., Amunts, A., and Ramakrishnan, V. (2017) The structure of the yeast mitochondrial ribosome, Science, 355, 528-531; doi: 10.1126/science.aal2415.

17. Waltz, F., Nguyen, T. T., Arrive, M., Bochler, A., Chicher, J., Hammann, P., Kuhn, L., Quadrado, M., Mireau, H., Hashem, Y., and Giege, P. (2019) Small is big in Arabidopsis mitochondrial ribosome, Nat. Plants, 5, 106-117; doi: 10.1038/s41477-018-0339-y.

18. Waltz, F., Soufari, H., Bochler, A., Giege, P., and Hashem, Y. (2019) Cryo-EM structure of the RNA-rich plant mitochondrial ribosome, bioRxiv; doi: 10.1101/777342.

19. Waltz, F., and Giege, P. (2019) Striking diversity of mitochondria-specific translation processes across eukaryotes, Trends Biochem. Sci., 45, 149-162; doi: 10.1016/j.tibs. 2019.10.004.

20. Ott, M., Amunts, A., and Brown, A. (2016) Organization and regulation of mitochondrial protein synthesis, Annu. Rev. Biochem., 85, 77-101; doi: 10.1146/annurev-biochem060815-014334.

21. Roll-Mecak, A., Shin, B. S., Dever, T. E., and Burley, S. K. (2001) Engaging the ribosome: universal IFs of translation, Trends Biochem. Sci., 26, 705-709.

22. Atkinson, G. C., Kuzmenko, A., Kamenski, P., Vysokikh, M. Y., Lakunina, V., Tankov, S., Smirnova, E., Soosaar, A., Tenson, T., and Hauryliuk, V. (2012) Evolutionary and genetic analyses of mitochondrial translation initiation factors identify the missing mitochondrial IF3 in $S$. cerevisiae, Nucleic Acids Res., 40, 6122-6134; doi: 10.1093/nar/ gks272.

23. Julian, P., Milon, P., Agirrezabala, X., Lasso, G., Gil, D., Rodnina, M. V., and Valle, M. (2011) The cryo-EM structure of a complete $30 \mathrm{~S}$ translation initiation complex from Escherichia coli, PLoS Biol., 9, e1001095; doi: 10.1371/ journal.pbio.1001095.

24. Myasnikov, A. G., Simonetti, A., Marzi, S., and Klaholz, B. P. (2009) Structure-function insights into prokaryotic and eukaryotic translation initiation, Curr. Opin. Struct. Biol., 19, 300-309.

25. Suzuki, T., Nagao, A., and Suzuki, T. (2011) Human mitochondrial tRNAs: biogenesis, function, structural aspects, and diseases, Annu. Rev. Genet., 45, 299-329; doi: 10.1146/annurev-genet-110410-132531.

26. Tan, T. H. P., Bochud-Allemann, N., Horn, E. K., and Schneider, A. (2002) Eukaryotic-type elongator tRNA ${ }^{\text {Met }}$ of Trypanosoma brucei becomes formylated after import into mitochondria, Proc. Natl. Acad. Sci. USA, 99, 1152-1157; doi: 10.1073/pnas.022522299.

27. Gaur, R., Grasso, D., Datta, P. P., Krishna, P. D., Das, G., Spencer, A., Agrawal, R. K., Spremulli, L., and Varshney, U. (2008) A single mammalian mitochondrial translation initiation factor functionally replaces two bacterial factors, Mol. Cell, 29, 180-190; doi: 10.1016/ j.molcel.2007.11.021.

28. Yassin, A. S., Haque, M. E., Datta, P. P., Elmore, K., Banavali, N. K., Spremulli, L. L., and Agrawal, R. K. (2011) Insertion domain within mammalian mitochondrial translation initiation factor 2 serves the role of eubacterial initiation factor 1, Proc. Natl. Acad. Sci. USA, 108, 39183923; doi: 10.1073/pnas.1017425108.

29. Kummer, E., Leibundgut, M., Rackham, O., Lee, R. G., Boehringer, D., Filipovska, A., and Ban, N. (2018) Unique features of mammalian mitochondrial translation initiation revealed by cryo-EM, Nature, 560, 263-267.

30. Vambutas, A., Ackerman, S. H., and Tzagoloff, A. (1991) Mitochondrial translational initiation and elongation factors in Saccharomyces cerevisiae, Eur. J. Biochem., 201, 643-652; doi: 10.1111/j.1432-1033.1991.tb16325.x.

31. Garofalo, C., Trinko, R., Kramer, G., Appling, D. R., and Hardesty, B. (2003) Purification and characterization of yeast mitochondrial initiation factor 2, Arch. Biochem. Biophys., 413, 243-252; doi: 10.1016/s0003-9861(03)00119-x.

32. Peske, F., Rodnina, M. V., and Wintermeyer, W. (2005) Sequence of steps in ribosome recycling as defined by kinetic analysis, Mol. Cell, 18, 403-412; doi: 10.1016/ j.molcel.2005.04.009.

33. Elvekrog, M. M., and Gonzalez, R. L. (2013) Conformational selection of translation initiation factor 3 signals proper substrate selection, Nat. Struct. Mol. Biol., 20, 628-633; doi: 10.1038/nsmb. 2554.

34. Valasek, L. S. (2012) "Ribozoomin" - translation initiation from the perspective of the ribosome-bound eukaryotic initiation factors (eIFs), Curr. Protein Pept. Sci., 13, 305-330.

35. Biou, V., Shu, F., and Ramakrishnan,V. (1995) X-ray crystallography shows that translational initiation factor IF3 consists of two compact alpha/beta domains linked by an alpha-helix, EMBO J., 14, 4056-4064; doi: 10.1002/j.14602075.1995.tb00077.x. 
36. Petrelli, D., La Teana, A., Garofalo, C., Spurio, R., Pon, C. L., and Gualerzi, C. O. (2001) Translation initiation factor IF3: two domains, five functions, one mechanism? EMBO J., 20, 4560-4569; doi: 10.1093/emboj/20.16.4560.

37. Koc, E. C., and Spremulli, L. L. (2002) Identification of mammalian mitochondrial translational initiation factor 3 and examination of its role in initiation complex formation with natural mRNAs, J. Biol. Chem., 277, 35541-35549; doi: 10.1074/jbc.M202498200.

38. Haque, M. E., and Spremulli, L. L. (2008) Roles of the $N$ and $C$-terminal domains of mammalian mitochondrial initiation factor 3 in protein biosynthesis, J. Mol. Biol., 384, 929-940; doi: 10.1016/j.jmb.2008.09.077.

39. Bhargava, K., and Spremulli, L. L. (2005) Role of the $N$ and $C$-terminal extensions on the activity of mammalian mitochondrial translational initiation factor 3, Nucleic Acids Res., 33, 7011-7018; doi: 10.1093/nar/gki1007.

40. Christian, B. E., and Spremulli, L. L. (2009) Evidence for an active role of IF3 $\mathrm{mt}$ in the initiation of translation in mammalian mitochondria, Biochemistry, 48, 3269-3278; doi: 10.1021/bi8023493.

41. Koripella, R. K., Sharma, M. R., Haque, M. E., Risteff, P., Spremulli, L. L., and Agrawal, R. K. (2019) Structure of human mitochondrial translation initiation factor 3 bound to the small ribosomal subunit, iScience, 12, 76-86; doi: 10.1016/j.isci.2018.12.030.

42. Chicherin, I. V., Zinina, V. V., Levitskiy, S. A., Serebryakova, M. V., and Kamenski, P. A. (2019) Aim23p interacts with the yeast mitochondrial ribosomal small subunit, Biochemistry (Moscow), 84, 40-46; doi: 10.1134/ S000629791901005X.

43. Levitskii, S., Derbikova, K., Baleva, M. V., Kuzmenko, A., Golovin, A. V., Chicherin, I., Krasheninnikov, I. A., and Kamenski, P. (2018) 60S dynamic state of bacterial ribosome is fixed by yeast mitochondrial initiation factor 3 , PeerJ., 6, e5620; doi: 10.7717/peerj.5620.

44. Derbikova, K., Kuzmenko, A., Levitskii, S., Klimontova, M., Chicherin, I., Baleva, M. V., Krasheninnikov, I. A., and Kamenski, P. (2018) Biological and evolutionary sig- nificance of terminal extensions of mitochondrial translation initiation factor 3, Int. J. Mol. Sci., 19; doi: 10.3390/ijms19123861.

45. Kuzmenko, A., Derbikova, K., Salvatori, R., Tankov, S., Atkinson, G. C., Tenson, T., Ott, M., Kamenski, P., and Hauryliuk, V. (2016) Aim-less translation: loss of Saccharomyces cerevisiae mitochondrial translation initiation factor mIF3/Aim23 leads to unbalanced protein synthesis, Sci. Rep., 6, 18749; doi: 10.1038/srep18749.

46. Herrmann, J. M., Woellhaf, M. W., and Bonnefoy, N. (2013) Control of protein synthesis in yeast mitochondria: the concept of translational activators, Biochim. Biophys. Acta, 1833, 286-294.

47. Derbikova, K. S., Levitsky, S. A., Chicherin, I. V., Vinogradova, E. N., and Kamenski, P. A. (2018) Activation of yeast mitochondrial translation: who is in charge? Biochemistry (Moscow), 83, 87-97.

48. Montoya, J., Ojala, D., and Attardi, G. (1981) Distinctive features of the 5 '-terminal sequences of the human mitochondrial mRNAs, Nature, 290, 465-470; doi: 10.1038/290465a0.

49. Weraarpachai, W., Antonicka, H., Sasarman, F., Seeger, J., Schrank, B., Kolesar, J. E., Lochmuller, H., Chevrette, M., Kaufman, B. A., Horvath, R., and Shoubridge, E. A. (2009) Mutation in TACO1, encoding a translational activator of COX I, results in cytochrome $c$ oxidase deficiency and late-onset Leigh syndrome, Nat. Genet., 41, 833-837; doi: 10.1038/ng.390.

50. Green-Willms, N. S., Butler, C. A., Dunstan, H. M., and Fox, T. D. (2001) Pet111p, an inner membrane-bound translational activator that limits expression of the Saccharomyces cerevisiae mitochondrial gene COX2, J. Biol. Chem., 276, 6392-6397; doi: 10.1074/jbc.M009856200.

51. Rudler, D. L., Hughes, L. A., Perks, K. L., Richman, T. R., Kuznetsova, I., Ermer, J. A., Abudulai, L. N., Shearwood, A. J., Viola, H. M., Hool, L. C., Siira, S. J., Rackham, O., and Filipovska, A. (2019) Fidelity of translation initiation is required for coordinated respiratory complex assembly, Sci. $A d v$., 5, eaay2118; doi: 10.1126/sciadv.aay2118. 